\title{
Estrategias de enseñanza en los entornos mediados: resultados de la experiencia de la performance virtual educativa
}

\section{Strategies of teaching in the mediated environments: results of the experience of the virtual educational performance}

\author{
Alejandro Ignacio Rodríguez Simon \\ Universidad Nacional de Educación a Distancia (UNED), Madrid, España \\ arodriguezsimon@invi.uned.es \\ Susana Regina López \\ Universidad Nacional de Quilmes (UNQ), Quilmes, Argentina \\ susana.lopez@unq.edu.ar
}

\begin{abstract}
Resumen
Se presenta aquí una experiencia de enseñanza y de aprendizaje que llamamos performance virtual basada en la teatralización de roles educativos desarrollada a través de encuentros sincrónicos en línea entre estudiantes, moderados por el equipo docente. Las performances educativas se relacionaron con contenidos sobre roles docentes y estereotipos en educación, donde parte del contenido programático de las asignaturas sirvió de fundamento para la búsqueda de las explicaciones asociadas al desarrollo técnico de las performances. La experiencia se desarrolló en la Universidad Nacional de Educación a Distancia (UNED) y en la Universidad Nacional de Quilmes (UNQ), en dos carreras de posgrado que se imparten a través de entornos mediados, y vinculadas a temas afines a la comunicación, a la educación y a la tecnología educativa. En este trabajo damos cuenta de los resultados de la experiencia de la performance virtual educativa en ambas universidades, a partir del análisis de datos recogidos a lo largo de las etapas de su puesta en marcha, que permiten caracterizarla como experiencia sincrónica innovadora en la enseñanza en los entornos virtuales.
\end{abstract}

Palabras clave

Entornos formativos virtuales, estrategias de enseñanza, performance educativa, innovación didáctica, escenificación mediada

\begin{abstract}
In this article, we present a teaching and learning experience that we call virtual performance based on the theatricalisation of educational roles developed through online synchronous meetings between students, moderated by the teaching team. The educational performances were related to content on teaching roles and stereotypes in education, where part of the programmatic content of the subjects served as a basis for the search for explanations associated with the technical development of the performances. The experience was developed in the Universidad Nacional de Educación a Distancia (UNED) and the Universidad Nacional de Quilmes (UNQ), in two postgraduate courses that are taught through virtual environments, and related to communication, to Education and to educational technology. This paper presents the results of the development of this virtual educational performance experience in both universities, from the analysis of data that was collected throughout the stages of its implementation, which allows to characterize it as an innovative synchronous experience in teaching in virtual environments.
\end{abstract}

Keywords

Virtual formative environments, teaching strategies, educational performance, educational innovation, mediated staging 


\section{Introducción}

Este trabajo presenta los resultados de una experiencia realizada en el marco de dos carreras de posgrado dictadas en modalidad no presencial con fuerte énfasis en el uso de recursos tecnológicos. La experiencia se basa en la puesta en marcha de una estrategia de enseñanza, enmarcada como innovación educativa, en la que se propuso a los estudiantes representar roles vinculados a las tareas de enseñanza y de aprendizaje en entornos virtuales en un encuentro sincrónico realizado a través del sistema de videoconferencias y/o chat del campus virtual de las universidades involucradas. La experiencia, entendida como una performance virtual educativa implicó, por parte de los estudiantes, la revisión bibliográfica de los contenidos de las asignaturas, la participación en foros por grupos para el acuerdo de los roles a representar, la puesta en común de motivaciones sobre la realización de la actividad, su desarrollo y su análisis a partir de registros de relatos orales y registros narrativos colaborativos.

\subsection{Objetivos}

En tanto experiencia sincrónica de puesta en escena de roles que tienen lugar cuando se desarrollan procesos de enseñanza y de aprendizaje en entornos virtuales, los objetivos de este trabajo son:

a. Presentar los resultados de la experiencia de performance virtual educativa en tanto estrategia de enseñanza innovadora en aulas virtuales.

b. Difundir la experiencia de performance virtual educativa entre el profesorado universitario preocupado por los procesos de enseñanza que se desarrollan en los entornos virtuales.

\section{Marco Teórico}

El desarrollo de las tecnologías ha implicado cambios en las relaciones sociales, comunicacionales y culturales (Álvarez y Morán, 2014). Distintos autores (Morales Almeida, 2016; Area Moreira, Fariña y González, 2013; Gros Salvat, 2011) sostienen que estos cambios se reflejan también en la educación y particularmente en los escenarios educativos donde las tecnologías resultan el soporte de comunicación e interacción para la enseñanza y el aprendizaje. En este marco, surgieron propuestas de educación mediadas por entornos tecnológicos donde el potencial está puesto en las posibilidades de mediación entre los sujetos, las tecnologías y el entorno, en tanto que una de las características de la educación a distancia consiste en la mediatización de las relaciones entre docentes y alumnos (Litwin, 2005).

Entender a las tecnologías como mediadoras implica otorgarle importancia a la construcción del conocimiento realizado por los estudiantes y las interacciones que se establecen a través de esta mediación. Así, el rol del docente resulta fundamental en tanto facilitador de esas mediaciones entre estudiantes y tecnologías, de modo que favorezca procesos de construcción de conocimiento horizontales y colaborativos, a través de la interacción entre todos los que participan de una propuesta de enseñanza (Aparici, 2010; Osuna, 2011, 2007).

Respecto del rol docente, la gestión de la enseñanza en los entornos mediados implica tomar una serie de decisiones didácticas, comunicacionales y tecnológicas, tales como 
organizar el plan de trabajo, planificar actividades de aprendizaje, proponer intercambios entre estudiantes, seleccionar la bibliografía, diseñar el aula virtual y organizar los materiales y recursos. Dichas decisiones, entendidas como estrategias, se toman desde un abanico de posibilidades disponibles y utilizadas por los profesores al momento del diseño, desarrollo y reflexión acerca de la enseñanza (Stenhouse, 1984). En consecuencia, las estrategias de enseñanza van de la mano de la autonomía del profesorado, en contraposición a la idea de método, en tanto reglas y preceptos a seguir: el profesor es capaz de tomar decisiones sobre la enseñanza de acuerdo con las situaciones y contexto en las que ésta tiene lugar. En otras palabras, las actividades que el profesor proponga no dependerán de estrategias únicas provenientes de un listado preestablecido, sino de las decisiones tomadas, los materiales y recursos, el agrupamiento de estudiantes, el entorno.

Desde esta perspectiva, las estrategias de enseñanza dan lugar a las innovaciones educativas que surgen cuando al interior de una institución educativa se planifican y se ponen en marcha acciones para lograr mejoras en las prácticas de enseñanza (Litwin, 2012). Pensar en innovaciones en la enseñanza requiere del estudio del contexto y de evaluar qué aspectos necesitan ser revisados y rediseñados. En términos de Litwin (2012) hablamos de la contextualización, la descontextualización y la recontextualización de una práctica innovadora.

Al hablar de innovaciones en la enseñanza conviene detenernos en dos aspectos: el primero es la relación entre las innovaciones y las tecnologías. Destacamos este aspecto ya que muchas veces se vincula la idea de innovación por la incorporación de tecnologías. Entendemos que el uso de las tecnologías no hace a una propuesta de enseñanza innovadora de por sí. Es el diseño didáctico y las decisiones ligadas a la enseñanza lo que definen a una propuesta como innovadora y no solamente por el uso de las tecnologías o su desarrollo en un contexto mediado.

El segundo aspecto que queremos destacar es que las innovaciones son contextualizadas. Eso significa que las innovaciones y las estrategias de enseñanza innovadoras no son transferibles de hecho o de forma automática. Una propuesta puede resultar innovadora en un contexto educativo, pero para replicarla en otro será necesario revisarla y recontextualizarla. Estos dos aspectos resultan centrales al momento de describir y posteriormente analizar la estrategia de enseñanza que presentamos en este artículo: la performance virtual. Pero antes vamos a presentar qué entendemos por performance y cuáles son sus cualidades al inscribirla como una propuesta educativa en un contexto virtual de enseñanza.

Sostenemos el concepto de performance como un concepto polisémico ligado a la idea de ejecución, realización, desempeño, así como también, actuación, escenificación, trama, acción, teatralización y puesta en escena (Turner, 2013; Szurmuk y McKee, 2009). Autores tales como Turner (2013) y Goffman (2012) sostienen que cualquier proceso social puede ser abordado en términos de performance teniendo en cuenta la estructura que posibilita las interacciones a través de las reglas establecidas en cada grupo social. El estudio de estas interacciones se realiza a través de la observación y descripción de las secuencias espacio temporales (Bianchotti y Ortecho, 2013; Schechner, 2000) en las que estos procesos se desarrollan. 
Ahora bien, el concepto de performance trasciende los distintos campos de estudio, en los que se encuentran la antropología, la sociología, la comunicación, el arte escénico y la educación, entre otros (Rodríguez Simon, 2017; Taylor y Fuentes, 2011). Distintas experiencias educativas (Álvarez y Bajardi, 2012; Vergara, 2009) muestran cómo las performances resultan estrategias valiosas para la puesta en escena y apropiación de contenidos a través de vivencias concretas, alejando a los estudiantes de la repetición y memorización de contenidos. Como ejemplo, mencionamos la experiencia desarrollada por Gómez Arcos (2005) en el que explica las posibilidades educativas de las performances en la enseñanza secundaria y el bachillerato para trabajar contenidos sobre lenguaje expresivo y el arte de acción, desde una perspectiva interdisciplinar, que incluye medios tecnológicos y multimedia. En esta experiencia, el autor señala como se han incluido contenidos para la edición de videos y fotografía para el desarrollo de las performances. Vemos entonces que al momento de diseñar la propuesta en una clase presencial se han considerado recursos tecnológicos para que los estudiantes documenten las teatralizaciones que desarrollaron. Este y otros antecedentes posibilitan la reflexión sobre el desarrollo de performances educativas en los entornos virtuales.

Como ya mencionamos, las tecnologías abren posibilidades educativas basadas en la interacción y comunicación mediadas que fomentan la participación horizontal, el trabajo colaborativo y el desarrollo de innovaciones sustentadas en supuestos pedagógicos. Es en este contexto en el que surge la propuesta de interacción que denominamos performance virtual educativa, que recupera todas las características propias de sus desarrollos previos en instancias tradicionales de educación, pero que se nutre de las posibilidades de interacción y colaboración entre pares al desarrollarse a través de los recursos que posibilitan las tecnologías en el aula virtual.

La construcción y el diseño de la performance virtual educativa considera los procesos que inicialmente no fueron pensados para la virtualidad, pero, aun así, es posible adaptarla a la modalidad de enseñanza en línea (Rodríguez Simon, 2017). En los próximos apartados describimos las características y el contexto de desarrollo de la performance virtual educativa en tanto estrategia de enseñanza para la puesta en escena y apropiación de contenidos sobre el rol de docentes y estudiantes en la enseñanza mediada.

\section{Descripción de la propuesta y contexto de desarrollo}

La experiencia de performance virtual educativa se implementó en el Máster en Comunicación y Educación en la Red de la Universidad Nacional de Educación a Distancia (UNED), España, y en la Especialización en Docencia en Entornos Virtuales de la Universidad Nacional de Quilmes (UNQ), Argentina, en las asignaturas "Educación y comunicación en el ciberespacio" (UNED) y "TIC y Enseñanza" durante los ciclos lectivos 2015/2016. Ambas comunidades educativas fueron seleccionadas de manera intencional, dado que comparten contenidos disciplinares, modos de acceso al conocimiento y promoción, por parte de los equipos docentes, de valores de cohesión y trabajo colaborativo.

Las plataformas educativas utilizadas por estas universidades, aLF en el caso de la UNED y Moodle en el caso de UNQ, resultaron las herramientas principales de interacción; dentro de ellas transcurrieron varias de las etapas de la propuesta. 
Pereira (2011) las define como plataformas "gótica" y "barroca", refiriéndose a aLF y Moodle respectivamente. La primera, determinada como plataforma compleja, con máximos niveles de seguridad y una optimización que resulta ideal en el manejo de amplios conglomerados de estudiantes y cursos, así como también comunidades de aprendizaje en línea. En cuanto a la segunda, está dotada de un nutrido repertorio de módulos y extensiones utilizables en el diseño de los cursos en línea, razón que la hace más versátil con respecto a aLF. Sin embargo, en lo que respecta a la configuración de permisos multiusuarios, administración y configuración de grupos, aLF se sitúa en un escalón más alto que su equivalente.

Para la realización de las actividades, ambas plataformas presentan características similares en lo que respecta a su uso para las experiencias vistas de manera global. Es de destacar que en ambas propuestas se utilizaron también aplicaciones externas al campus virtual. Esta decisión se fundamentó en la necesidad, en las dos asignaturas de promover la experimentación de distintos recursos y posibilidades de la Web 2.0.

En cuanto a la participación total de estudiantes en cada una de las asignaturas puede verse en el siguiente cuadro:

\begin{tabular}{|c|c|c|}
\hline & $\begin{array}{c}\text { Universidad Nacional de } \\
\text { Educación a Distancia } \\
\text { (UNED) - España }\end{array}$ & $\begin{array}{c}\text { Universidad Nacional } \\
\text { de Quilmes } \\
\text { (UNQ) - Argentina }\end{array}$ \\
\hline Asignatura & $\begin{array}{c}\text { Educación y Comunicación } \\
\text { en el Ciberespacio }\end{array}$ & TIC y Enseñanza \\
\hline Año lectivo & Curso 2015/2016 & Curso 2016 \\
\hline $\begin{array}{c}\text { Cantidad de estudiantes } \\
\text { participantes (total: 123) }\end{array}$ & 83 & 40 \\
\hline
\end{tabular}

Tabla 1: Distribución de estudiantes según universidad y asignaturas. Fuente: Elaboración propia.

Forman parte del programa curricular de ambas asignaturas contenidos vinculados al aprendizaje, la cognición y el ejercicio del rol docente en la enseñanza mediada por tecnologías. Entre sus objetivos, las asignaturas se proponen promover el uso de herramientas y dispositivos tecnológicos en las aulas virtuales como mediadores de las actividades de enseñanza, de aprendizaje y de evaluación. Teniendo en cuenta estos objetivos, y entendiendo que la enseñanza en un aula virtual debe propiciar espacios de interacción, colaboración y puesta en acción de los contenidos a través de los recursos del aula, diseñamos la experiencia de la performance virtual cuyos propósitos fueron (Rodríguez Simon, 2017):

- Seleccionar un rol característico de la formación en entornos virtuales para representarlo en una interacción grupal mediada por tecnologías.

- Representar los roles enmarcados en escenas para caracterizarlos.

- Experimentar la interacción en la construcción de una realidad educativa a través de una videoconferencia o un chat. 
- Conocer los diferentes roles intervinientes en los procesos de enseñanza y de aprendizaje a través de la interacción desarrollada a través de las performances.

- Analizar la acción performativa de estos roles y presentar sus reflexiones a través de relatos orales y narraciones colectivas.

La experiencia implicó distintas etapas: una etapa preparatoria, una etapa de puesta en escena y una etapa de reflexión final.

En la etapa preparatoria se presentó a los estudiantes la consigna de la performance virtual educativa. Se dividió a los estudiantes en grupos y se le facilitó a cada grupo un foro. Se le solicitó a cada estudiante que, una vez comprendida la bibliografía, eligiera un rol vinculado a la enseñanza en los entornos virtuales, debiéndolo comunicar en el foro del grupo al que pertenecía. En el mensaje debían indicar el rol seleccionado para representar, sus características, fundamentar la elección y acordar una fecha para desarrollar la performance en un encuentro virtual sincrónico. El hecho de que todos los integrantes del grupo vieran las características de los roles elegidos por sus compañeros con anterioridad al encuentro sincrónico, facilitó el desenvolvimiento de la propuesta. Esto se dio de esta manera ya que cada participante pudo con antelación, contar con un panorama de los roles con los que iba a interactuar en la instancia de la performance.

Entre los roles elegidos, mencionamos a modo de ejemplo, estudiante impulsivo, profesor "conductista", profesor "constructivista", estudiante sensible y curioso, tutor virtual, estudiante reflexivo, estudiante muy participativo, profesor "inabordable", entre muchos otros propuestos por los alumnos. Al momento de presentar las consignas de trabajo y durante el proceso de selección de roles a representar, los estudiantes plasmaron sus inquietudes respecto de la actividad en un foro, antes al desarrollo de la performance.

En la etapa de puesta en escena tuvo lugar la performance virtual propiamente dicha. Los estudiantes, moderados por el equipo docente de las asignaturas se encontraron sincrónicamente en las fechas y horarios pautados por cada grupo a través del chat o el sistema de videoconferencias del campus virtual. Esto implicó un cambio habitual en la dinámica de estas materias, ya que la comunicación se da habitualmente por recursos que permiten la comunicación asincrónica.

Para facilitar el intercambio y como pretexto para que cada estudiante pusiera en escena el rol elegido, se les presentó una noticia educativa de actualidad y cada uno debió opinar e interactuar desde el rol elegido. Las interacciones fueron variables en tanto que oscilaron entre performances que duraron entre 30 a 60 minutos aproximadamente. Cabe destacar que, en este tiempo de interacción, se dedicaron unos minutos iniciales a configurar los recursos y verificar que todos los alumnos tuvieran las condiciones tecnológicas adecuadas que les permitieran la participación de la performance virtual.

En cuanto a las estrategias de moderación por parte de los docentes, éstos se limitaron a explicar el ejercicio que incluía la lectura de una noticia educativa para generar un debate desde la postura del rol elegido. Los docentes determinaron también el comienzo de la representación, ciñéndose a intervenir de manera esporádica y decidir cuándo concluía la secuencia de actuación para luego culminar con la moderación de un debate final de carácter autoreflexivo por parte de los estudiantes. 
Durante estos minutos iniciales se les pidió autorización para grabar las interacciones cuando fueron desarrolladas mediante videoconferencias o guardar los registros de las conversaciones de aquellas performances desarrolladas a través de chats. Una vez cerrada la puesta en escena de los roles elegidos, e inmediatamente finalizados los intercambios, se les solicitó a los alumnos que comentaran sus impresiones sobre los roles representados y la experiencia desarrollada. El equipo docente de las asignaturas dispuso una serie de consignas para abrir la reflexión sobre la experiencia, dejando que los estudiantes expresaran sus impresiones sobre la tarea realizada. Las impresiones fueron registradas mediante el software a través del cual se realizó la videoconferencia donde cada estudiante comentó al grupo su vivencia y contraste entre la experiencia y sus inquietudes o motivaciones iniciales. En el caso de las performances realizadas mediante chat, el registro se realizó a través del resguardo de la transcripción de los mismos.

En la etapa final de reflexión, se les solicitó a los estudiantes como actividad de entrega obligatoria, la edición de una narración colectiva en el aula virtual, que reflejara las impresiones grupales sobre la experiencia, la actuación a partir del rol elegido, su puesta en escena y fundamentalmente, en qué medida la experiencia les permitió articular la bibliografía de las asignaturas con las posibilidades de pensar sobre de la importancia de considerar al "otro" en el diseño de instancias de formación mediadas por tecnologías.

En el siguiente cuadro, se presentan los instrumentos de recogida de datos en relación al tipo de información a recabar en cada etapa: inquietudes iniciales, impresiones inmediatamente luego de finalizar las performances y reflexión final de cierre de la experiencia:

\begin{tabular}{|c|c|c|c|}
\hline $\begin{array}{c}\text { Etapas de } \\
\text { desarrollo de la } \\
\text { Performance }\end{array}$ & $\begin{array}{c}\text { Objetivos de cada } \\
\text { etapa }\end{array}$ & $\begin{array}{c}\text { Instrumento } \\
\text { implementado y } \\
\text { contexto participativo }\end{array}$ & Tipo de reflexión \\
\hline $\begin{array}{l}\text { Etapa } \\
\text { preparatoria }\end{array}$ & $\begin{array}{lr}\text { Recolección } & \text { de } \\
\text { inquietudes } & \text { y } \\
\text { motivaciones frente } \\
\text { a propuesta } & \text { de } \\
\text { encuentro } & \\
\text { sincrónico } & \text { de } \\
\text { teatralización } & \text { del } \\
\text { rol elegido } & \text { para } \\
\text { representar } & \text { los } \\
\text { procesos } & \text { de } \\
\text { enseñanza y } & \text { de } \\
\text { aprendizaje } & \text { en } \\
\text { entornos virtuales }\end{array}$ & $\begin{array}{l}\text { Participación narrativa } \\
\text { asincrónica en un foro } \\
\text { por grupos en el aula } \\
\text { virtual }\end{array}$ & $\begin{array}{l}\text { Aporte individual } \\
\text { en foro grupal }\end{array}$ \\
\hline $\begin{array}{l}\text { Etapa de } \\
\text { puesta en } \\
\text { escena }\end{array}$ & $\begin{array}{l}\text { Recolección de } \\
\text { impresiones } \\
\text { posteriores }\end{array}$ & \begin{tabular}{lr} 
Relatos & \multicolumn{2}{r}{ registrados } \\
mediante & el software \\
utilizado & para el
\end{tabular} & $\begin{array}{ll}\text { Aporte } & \text { individual } \\
\text { en } & \text { encuentro } \\
\text { grupal } & \text { sincrónico }\end{array}$ \\
\hline
\end{tabular}

Estrategias de enseñanza en los entornos mediados: resultados de la experiencia de la performance virtual educativa. Alejandro Rodríguez Simon y Susana Regina López. 


\begin{tabular}{|c|c|c|c|}
\hline & $\begin{array}{l}\text { inmediatamente al } \\
\text { finalizar las } \\
\text { teatralizaciones } \\
\text { (performances) }\end{array}$ & $\begin{array}{ll}\text { desarrollo de las } \\
\text { videoconferencias } & \text { y/o } \\
\text { chat } & \end{array}$ & $\begin{array}{l}\text { mediante } \\
\text { videoconferencias } \\
\text { y/o chat }\end{array}$ \\
\hline $\begin{array}{l}\text { Etapa final } \\
\text { de reflexión }\end{array}$ & $\begin{array}{l}\text { Recolección de } \\
\text { reflexiones por } \\
\text { grupos sobre las } \\
\text { performances de } \\
\text { acuerdo con pautas } \\
\text { establecidas por el } \\
\text { equipo docente de } \\
\text { las asignaturas }\end{array}$ & $\begin{array}{lr}\text { Relato } & \text { narrativo } \\
\text { realizado de } & \text { manera } \\
\text { colaborativa } & \end{array}$ & $\begin{array}{l}\text { Aporte grupal de } \\
\text { construcción } \\
\text { colaborativa en } \\
\text { wiki o documento } \\
\text { colectivo }\end{array}$ \\
\hline
\end{tabular}

Tabla 2: Instrumentos de recogida de datos implementados en cada etapa de las performances. Fuente: Elaboración propia.

Seguidamente presentamos el análisis que se realizó a partir de la información recogida en cada una de las etapas de la experiencia.

\section{Análisis de la experiencia}

Una vez finalizada la experiencia y aplicados los instrumentos de recogida de datos, tuvo lugar el proceso de análisis en función de los objetivos propuestos. El análisis, de carácter cualitativo, se realizó siguiendo un criterio temático para la identificación de aportes que mencionaban reflexiones comunes, encontrando así segmentos que hablaban sobre los mismos temas. Se describen a continuación estos núcleos temáticos y se incluyen algunas respuestas que permiten ejemplificarlos.

\section{a) Expectativas iniciales ante la propuesta}

La publicación de las consignas de la experiencia provocó expectativas e incertidumbre en los estudiantes en ambas asignaturas. Estas expectativas fueron provocadas por dos motivos: el primero por ser encuentros sincrónicos, en tanto que el modelo de ambas propuestas formativas es mayormente asincrónico. El segundo, porque la experiencia provocó expectativas ya que movilizó de su rol tradicional, sacándolos de su zona de confort (Luckner y Nadler, 1997) de estudiante receptor y los ubicó en el lugar de la acción, con fuerte énfasis en la oralidad y el intercambio "cara a cara". Algunos ejemplos que ilustran estas expectativas son:

"Nerviosa por el desconocimiento de la actividad y por el hecho de dejarme ver en un entorno en el que hasta ahora estabas detrás de una pantalla, oculta, y sólo te dejabas ver mediante el lenguaje escrito. Mucho más pausado y menos espontáneo." (alumna 52, UNED).

"Tengo miedo de enfrentarme cara a cara con mis compañeros. Será la primera vez, ya veremos qué sale." (alumno $1, U N Q$ ).

"Yo elegí el rol de alumno impulsivo, pero en realidad soy una persona tímida. Por esta razón, estoy expectante y ansioso por que hagamos esta experiencia. ¿Qué va a pasar?" (alumno 7, UNQ). 
"(...) intentar jugar un rol, con todo lo que esto implica, tratando de vencer la timidez y la vergüenza propia que tenemos los adultos." (alumno 92, UNED).

\section{b) Reconocimiento del otro en la enseñanza en los entornos virtuales}

Uno de los objetivos de la experiencia fue que los estudiantes pudieran conocer a través de la vivencia desarrollada en las performances distintas situaciones que tienen lugar en la interacción que se da en los procesos de enseñanza y de aprendizaje mediados por tecnologías, en tanto que estos aspectos forman parte de los contenidos del programa curricular de ambas asignaturas. En este sentido, lejos de presentar estos contenidos de manera lineal, se optó por presentar la bibliografía y posteriormente articularla con la experiencia concreta de lo que sucede en los intercambios en línea entre docentes y estudiantes y estudiantes entre sí. Comprender sobre la moderación y las posibilidades de ubicarse en el lugar del otro en los procesos de enseñanza mediados por tecnologías resultó uno de los aprendizajes que los estudiantes manifiestan a partir de esta experiencia. Los siguientes fragmentos recuperados de las reflexiones posteriores a la realización de las performances y de las reflexiones finales, son ejemplos de estas apropiaciones:

"Mezclar un modelo pedagógico con juegos de simulación no sólo fue útil sino revelador para comprender las dimensiones de la categoría "rol", y cómo juegan los contextos, las particularidades y las subjetividades que se ponen en juego en el momento de la interacción en situaciones de enseñanza aprendizaje." (alumna 42, $U N Q)$.

"Rescato varios aspectos positivos. Entre ellos (...) conocer la perspectiva del otro, es decir, el paradigma desde el cual se posiciona para expresar su punto de vista y sensaciones, y saber que está, que no es un robot ni nada por el estilo, sino alguien como yo, que también tiene sueños, deseos y expectativas." (alumna 18, UNQ).

"Ha sido una experiencia significativa ya que nos dio la posibilidad de ponernos en el lugar del otro (el personaje). Cuando en nuestras aulas elaboramos actividades en la mayoría de los casos no tuvimos en cuenta a la diversidad. En ella se encuentran los diversos personajes: alumnos y docentes." (alumna 32, $U N Q$ ).

"Lo bueno de esta experiencia ponerse en el lugar del alumno, qué le pasa, qué siente; ver los rostros humaniza: cuando veo, me identifico con el otro." (alumno 25, $U N Q)$.

"Me sirvió para entender los nervios, ansiedad y dificultades que pueden experimentar los alumnos en este tipo de encuentros." (alumno 3, UNQ).

"A lo largo de la experiencia de roles, se pudo resumir todo lo trabajado a lo largo de la cursada. Realizar aportes personales y además al salir del espacio de confort, pudimos situarnos desde el lugar del otro y entender la amplitud de posibilidades que ofrece la educación virtual." (alumna 27, UNQ).

\section{c) Apropiación de contenidos a través de la articulación práctica}

En relación a la apropiación de los contenidos programáticos por parte de los estudiantes, ambas asignaturas se orientan a la articulación entre la teoría y la práctica, intentando favorecer la construcción colaborativa entre los alumnos y alejar al equipo 
docente de la mera transmisión de contenidos. Esta premisa fue percibida por los estudiantes, expresándolo en los siguientes comentarios:

"El aula interactiva nos permitió el intercambio de ideas a partir de la temática propuesta, lo que significó un entorno reflexivo a partir del cual pudimos sacar información relevante." (alumna 7, UNQ).

"La experiencia fue muy interesante. Creo que ponerse en el lugar del otro, es la parte más difícil. Tenemos que tratar de entender qué le pasa al otro, con sus miedos, inquietudes y expectativas, para lograr sus objetivos en la simulación. Para lograr esos objetivos se tienen que resolver distintas situaciones y problemas que se plantean. Se vive lo que se quiere aprender y no solamente se piensa en ello." (alumna 27, UNQ).

"La experiencia desarrollada nos permite, también, recuperar los aportes teóricos trabajados en el módulo en relación a los procesos comunicativos que de despliegan en entornos virtuales; por un lado, las contribuciones de Jean Cloutier (1973) quien propone su teoría sobre "emirec", es decir de sujetos tanto emisores como receptores de diversos mensajes y a sus propuestas de Alvin Tofler (1980) al señalar el término "prosumidor", esto es productores y consumidores de información. Lo señalado resulta de gran importancia en cuanto a los procesos comunicativos donde se revisan los roles preasignados a los actores educativos, así por ejemplo es el o los docentes quien tradicionalmente tenían la tarea de transmitir la información, eran la fuente del conocimiento." (alumna 89, UNED).

"Ahora bien, en entornos virtuales de aprendizaje, este modelo estático de la comunicación requiere ser revisado. Tal fue el caso de la Videoconferencia llevada a cabo en el grupo, donde existían roles preestablecidos pero el resultado del proceso comunicativo era coral, se presentaba voces cada uno desde su ámbito y sus aportes favorecían o no el desarrollo de la actividad." (alumna 11, UNQ).

\section{d) Valoración de la comunicación sincrónica}

Uno de los aspectos que más interpeló a los estudiantes al momento de presentar los contenidos fue la posibilidad de realizar un encuentro sincrónico, ya que los modelos de enseñanza de ambas propuestas formativas están basados predominantemente en la asincronía. La posibilidad de interactuar con el equipo docente y con los compañeros en tiempo real fue valorado por los estudiantes al experimentar durante la cursada de las asignaturas una variedad de recursos de interacción. Así lo reflejan las siguientes reflexiones:

"Rescato varios aspectos positivos. Entre ellos (...) la necesidad de comunicarnos, de acortar las distancias geográficas, y a través de un pequeño recuadro, iconocernos, vernos y escucharnos!” (alumna 18, UNQ).

"La experiencia nos hace darnos cuenta del potencial de los recursos tecnológicos, en particular de la videoconferencia, la cual no sólo permitió que podamos realizar el juego de roles, sino que, además, y fundamentalmente, nos dio la posibilidad de conocernos, vernos las caras y escucharnos las voces. También nos dimos cuenta de los problemas que pueden surgir durante el uso de las tecnologías, que pueden fallar, total o parcialmente, del punto de vista técnico." (alumna 22, UNQ). 
"Me gustó la experiencia, (...) lo más enriquecedor, sin dudas, fue el intercambio y la empatía con los otros participantes.” (alumno 9, UNQ).

"Al principio tenía reticencias a hacer el hangout, por la vergüenza de verte y de que los demás te vean y encima "actuando". Pero una vez realizado, ha merecido la pena, por varios motivos. Lo primero es que nos hemos visto, y es que cuando estás realizando trabajos con personas que están al otro lado del ordenador y no les conoces a veces piensas que "estás trabajando con robots". Y gracias a esta representación nos hemos visto y conocido, aunque haya sido durante un breve espacio de tiempo, "ya tenemos cara". (alumno 73, UNED).

"Ha sido un éxito y animaría al resto de compañeros a que realizaran esta tarea con video conferencia, porque aporta: gestos, entonación y todo tipo de acciones que aporta muchísima connotación al ejercicio." (alumno 122, UNED).

"Durante esta actividad no he podido olvidarme de la película "The Breakfast Club" ("El Club de los Cinco" en España) en la que un grupo de cinco alumnos de un instituto se ven obligados a mostrarse y a conocerse. Los roles, los personajes van cayendo y afloran ricas e interesantes personalidades, dramas y amistades. Añadir la imagen a esta actividad nos ha permitido además desvirtualizarnos parcialmente. Es agradable poner cara a los compañeros, alumnos y profesores, de un máster tan intenso como este." (alumno 64, UNED).

\section{e) Identificación de la propuesta de enseñanza como novedosa}

Retomando la idea de innovación educativa expuesta en el marco conceptual de este trabajo, entendemos a las propuestas de enseñanza enmarcadas como innovadoras a aquellas que hacen hincapié en su diseño didáctico y no solo en los aspectos tecnológicos. Esto fue percibido por los estudiantes, valorando la actividad como novedosa por los recursos tecnológicos implementados, pero también por la propuesta en sí, tal como lo expresan en los siguientes comentarios:

"Quedé contenta con el encuentro sincrónico que mantuve ayer con mis compañeros del grupo 4. Una experiencia totalmente novedosa, al menos para para mí, que jamás había participado de una actividad de esta naturaleza. Más allá de algunos inconvenientes técnicos que tuvimos que sortear, ilogramos concretar la comunicación! Mediada por la tecnología, por supuesto, pero comunicación al fin...” (alumna 14, UNQ).

"La verdad que a mí me dejo una sensación hermosa. Si bien trabajo con estas tecnologías cotidianamente, jamás como una docente intentando que un alumno vea que utilizar estas tecnologías le permite acceder a los materiales en cualquier momento. Como así también generador de su propio aprendizaje guiado por el docente y/o tutor. (...) Me encanto la experiencia... Siempre es bueno sacar provecho y críticas para mejorar la práctica diaria... Seguimos..." (alumna 27, $U N Q)$.

"Me resulto muy enriquecedor primero por el hecho de utilizar medios virtuales nuevos para mí, los cuales no estoy habituado a usarlos para realizar una actividad grupal. Durante el dialogo de mis compañeros que representaban uno con el rol de docente conductista y otro con el rol de alumno tecnológico o virtual y el mío de alumno tradicional, pude interpretar e interactuar a parte de mi rol e

Estrategias de enseñanza en los entornos mediados: resultados de la experiencia de la performance virtual educativa. Alejandro Rodríguez Simon y Susana Regina López. 
imaginar el rol de mis compañeros tratando desde cada actuación que desarrollamos lograr la comunicación e interacción entre pares y así llegar al aprendizaje." (alumno 10, UNQ).

\section{Conclusiones}

De la experiencia realizada se desprende que generó expectativas iniciales entre los estudiantes en tanto experiencia novedosa, no sólo por la posibilidad de participar de manera síncrona mediante chat o videoconferencias, sino por tener que pensar en la construcción de un rol y sostener su representación en la teatralización grupal.

La experiencia permitió abordar los contenidos de ambas asignaturas articulando los aspectos teóricos con la vivencia de los roles que tienen lugar en la enseñanza en entornos virtuales. Poder ponerse en el lugar del otro y experimentar a través de la práctica todas aquellas interrelaciones que se dan en la enseñanza mediada entre los distintos actores que intervienen, fue un aspecto valorado por los estudiantes. Destacamos que entre las dos universidades en las que llevamos adelante la experiencia de la performance virtual educativa, no hubo diferencias significativas al momento de su desarrollo, identificando los dos grupos a la experiencia como innovadora o "fuera de lo común."

Respecto de las innovaciones en la enseñanza, expresamos al inicio de este trabajo las evaluaciones y adaptaciones necesarias que hay que realizar si queremos replicar una propuesta en un nuevo contexto, de modo que la innovación genere cambios y mejoras. En este sentido, consideramos que será necesario un estudio previo sobre el contexto educativo y el modelo de enseñanza con el uso de tecnologías, antes de replicar esta experiencia en otra propuesta formativa de otra institución de educación superior.

Se nos abren diferentes interrogantes a partir de la puesta en marcha de la experiencia y a la vista de los resultados. Creemos que se pueden realizar variantes de las performances en donde el docente interactúe, pero acentuando aún más su rol de mediador, donde el 'dejar hacer' sea más posible. Por esta razón, pensamos que pueden surgir experiencias de performances que podríamos llamar ensambladas o sincrónicas sin intervención docente en su puesta en escena. En ellas, los alumnos podrán experimentar la co-creación y la puesta colectiva a través de la acción performativa. El ejercicio permitirá en este caso también, una reflexión sobre el reconocimiento del otro, como par, y sobre las alternativas de salida de las zonas estancas de la educación.

Los estudiantes venían cursando generalmente de manera asíncrona, por ello la propuesta de la actividad, independientemente de usar chat o videoconferencia, los desplazaba de su zona de confort. En ambas modalidades, salir de la zona cómoda y llegar a la zona de aprendizaje implicó el paso por las tres etapas anteriormente descriptas._Como punto de diferencia entre las experiencias acontecidas entre chat y videoconferencia, se reflejó el escudarse y soltarse en el primero, y no tanto en el segundo donde debieron superar el pánico escénico de encontrarse cara a cara con sus compañeros o con el docente. De todas formas, la mayor parte del estudiantado de ambas universidades lograron superar sus miedos, no influyendo en la mayoría de los casos si se trataba de uno u otro medio de interacción sincrónica. Podemos agregar sí, 
que los miedos se superaron antes en el chat durante la representación y en el caso de videoconferencia se produjo más bien en la reflexión.

Estas cuestiones nos permiten continuar la reflexión para seguir pensando en el rediseño y planificación de propuestas para la innovación de la enseñanza en los entornos virtuales en la educación superior.

Presentación del artículo: 3 de octubre de 2017 Fecha de aprobación: 29 de noviembre de 2017 Fecha de publicación: 22 de diciembre de 2017

Rodríguez Simon, A y López, S. (2017). Estrategias de enseñanza en los entornos mediados: resultados de la experiencia de la performance virtual educativa. RED, Revista de Educación a Distancia. 55. Recuperado de: http://www.um.es/ead/red/55/ rodriguez_lopez.pdf

\section{Financiación}

Esta investigación no ha recibido ninguna subvención específica de los organismos de financiación en los sectores públicos, comerciales o sin fines de lucro.

\section{Bibliografía}

Álvarez, D., \& Bajardi, A. (2012). La performance como experiencia educativa en secundaria. Arte, educación y cultura. Aportaciones desde la periferia. Jaén: Colegio Oficial Bellas Artes Sevilla.

Álvarez, G., \& Morán, L. (2014). ¿Cómo se dispone a los docentes para futuras prácticas con tecnologías? Análisis sobre la inclusión tecnológica en cursos de formación. RED. Revista de educación a distancia, 43, 1-16.

Aparici, R. (2010). (coord.) Conectados en el ciberespacio. Madrid: UNED.

Area Moreira, M., Fariña, E., \& González, C. (2013). ¿Qué uso hacen de las aulas virtuales los docentes universitarios? RED. Revista de educación a distancia, 35, $1-13$.

Bianchotti, M., \& Ortecho, M. (2013). La noción de performance y su potencialidad epistemológica en el hacer científico social contemporáneo. Tabula Rasa, 19, 119-137.

Goffman, E. (2012). La presentación de la persona en la vida cotidiana. Buenos Aires: Amorrortu.

Gómez Arcos, J. R. (2005). Posibilidades educativas de la performance en la enseñanza secundaria. Arte, individuo y sociedad, 17, 115-132.

Gros Salvat, B. (2011). Evolución y retos de la educación virtual. Construyendo el elearning del siglo XXI. Barcelona: UOC.

Litwin, E. (2005). La educación a distancia: temas para el debate en una nueva agenda educativa. Buenos Aires: Amorrortu. 
Litwin, E. (2012). El oficio de enseñar. Condiciones y textos. Buenos Aires: Paidós.

Luckner, J., \& Nadler, R. (1997). Processing the experience. Strategies to Enhance and Generalize Learning. Dubuque, Iowa: Kendall / Hunt Publishing Company.

Morales Almeida, P. (2016). La formación del profesorado ante las TIC. Textos. Revista internacional de aprendizaje y cibersociedad, 17(1), 47-58.

Osuna, S. (2007). configuración y gestión de plataformas virtuales. Programa modular en tecnologías digitales y sociedad del conocimiento. Madrid: UNED.

Osuna, S. (2011). Aprender en la Web 2.0. Aprendizaje colaborativo en comunidades virtuales. La educación. 145, 1-19.

Pereira, J. (2011). Plataformas educativas libres. Recuperado de http://tallercomunicacionyeducacion.files.wordpress.com/2011/05/plataformas_e ducativas_libres_jesus_pereira.pdf

Rodríguez Simon, A. (2017). Performances y rituales en los procesos de enseñanza virtual (tesis doctoral). Madrid: Universidad Nacional de Educación a Distancia.

Schechner, R. (2000). Performance: Teoría y Prácticas Interculturales. Buenos Aires: Libros del Rojas y Universidad de Buenos Aires.

Stenhouse, L. (1984). Investigación y desarrollo del curriculum. Madrid: Morata.

Taylor, D., \& Fuentes, M. (2011). Estudios avanzados de performance. México: Bixio.

Turner, V. (2013). La selva de los símbolos. México: Siglo XXI.

Vergara, J. (s.f.). La performance como recurso didáctico. Recuperado de http://es.calameo.com/read/0010444721c5e2e9e9f12.

Estrategias de enseñanza en los entornos mediados: resultados de la experiencia de la performance virtual educativa. Alejandro Rodríguez Simon y Susana Regina López. 